P. Berlit ${ }^{1}$, F. Erbguth ${ }^{2}$, R. Hagenah ${ }^{3}$, U. Meyding-Lamadé ${ }^{4}$, C. Mohs ${ }^{5}$, T. Thiekötter ${ }^{1}$

${ }^{1}$ Deutsche Gesellschaft für Neurologie, Berlin, Deutschland

${ }^{2}$ Klinik für Neurologie, Universitätsklinik der Paracelsus Medizinischen Privatuniversität, Nürnberg, Deutschland

${ }^{3}$ Rotenburg/Wümme, Deutschland

${ }^{4}$ Neurologische Abteilung, Krankenhaus Nordwest, Frankfurt am Main, Deutschland

${ }^{5}$ Frankfurt am Main, Deutschland

\title{
Rudolf Wilhelm Christian Janzen
}

\subsubsection{0-15.07.2019}

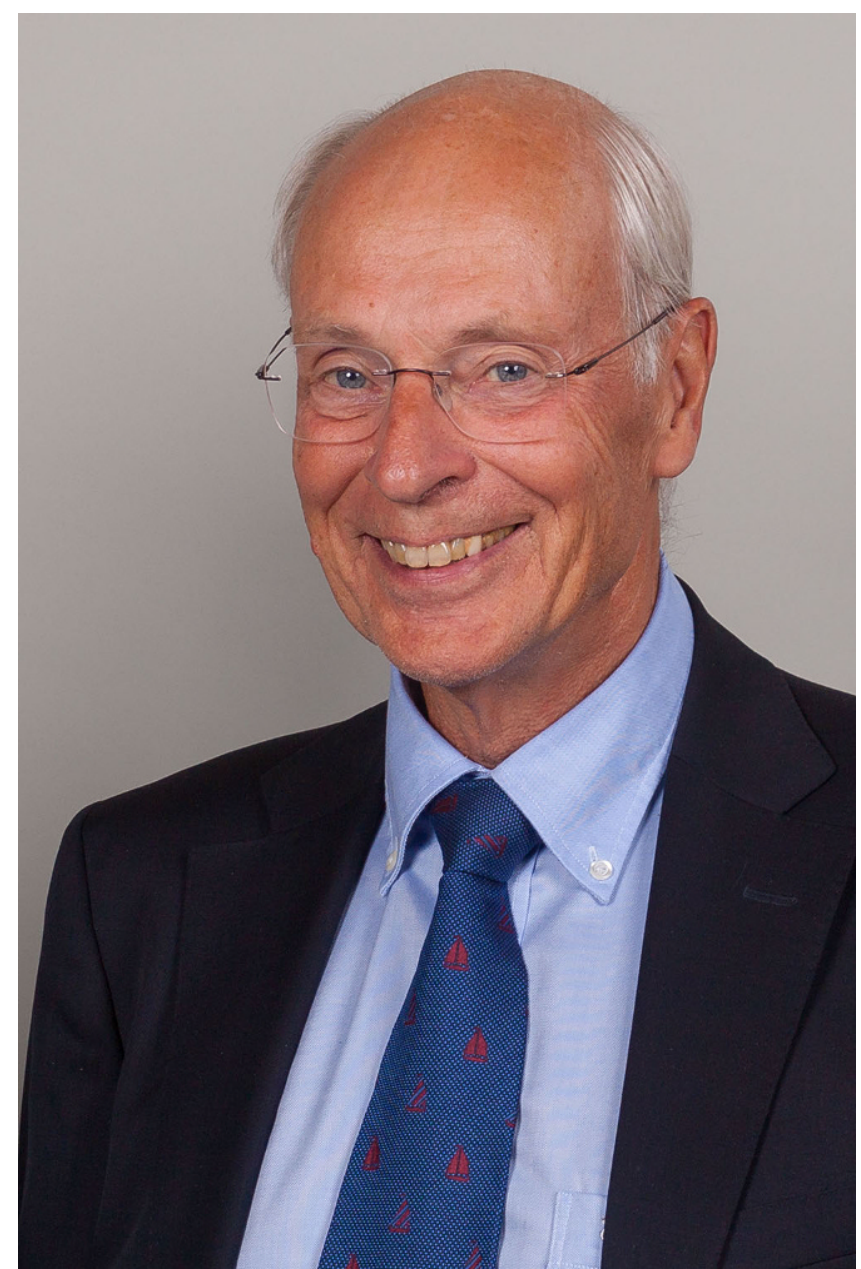

Rudolf Wilhelm Christian Janzen

Traurig und fassungslos sind wir alle - ein tragischer Unfall hat Prof. Dr. med. Rudolf C. Janzen am 15.07.2019 aus dem vollen Leben gerissen. Wir erinnern ihn als lebhaften Diskutanten, alles und alle - auch immer sich selbst - noch einmal in Frage stellend, mit klugen Gedanken, querdenkend, offen, kritisch

DGNeurologie 2019 · 2 (6): 531-532

https://doi.org/10.1007/s42451-019-00125-0

Online publiziert: 16. Oktober 2019

(c) Springer Medizin Verlag GmbH, ein Teil von Springer Nature und immer voller Respekt und Zuneigung seinen Mitmenschen gegenüber.

Prof. Dr. Rudolf C. Janzen war einer der großen Neurologen unserer Zeit. Schon als Student wie auch als junger Assistenzarzt bis hin in den Ruhestand begeisterte er sich für das Fachgebiet Neurologie. Er war fasziniert von der Struktur und Funktion des Gehirns. Seit 1980 war er Mitglied der DGN (Deutsche Gesellschaft für Neurologie).

"Die Liebe ist die Tochter der Erkenntnis, die Liebe ist umso glühender, je tiefer die Erkenntnis ist." (Leonardo da Vinci, 1452-1519)

Dieser Satz traf auf Prof. Dr. Rudolf C. Janzen zu - sowohl im privaten als auch im beruflichen Leben, der Neurologie.

Nach dem Studium in Münster, Berlin und Zürich folgte die Medizinalassistentenzeit in der Neuroanatomie in Hamburg, in der Frauenheilkunde in Dortmund, in der Neurochirurgie in Bochum-Langendreer, in der Pathologie in Nürnberg sowie in der Inneren Medizin im Asklepios Klinikum Harburg in Hamburg. Von 1969-1971 arbeitete er als Assistent in der Neurophysiologie in Münster. Von 1971 an arbeitete er an der Neurologischen Universitätsklinik Eppendorf in Hamburg. Hohe Erwartungen waren an den fleißigen, umtriebigen, jungen Wissenschaftler gestellt, besonders von seinem Vater, dem neurologischen Ordinarius der Neurologischen Universitätsklinik Hamburg-Eppendorf, den er bis zu dessen Tod verehrte. 1977 habilitierte er sich mit dem Thema „Krampfbegleitende Motorik" an der Universität Hamburg.

Er wurde 1985 an die Neurologische Klinik des Krankenhauses Nordwest in Frankfurt berufen, eine der großen und renommierten, nichtuniversitären Kliniken für Neurologie in Deutschland. Hier prägte er 20 Jahre die Geschicke dieser Klinik als Chefarzt und einige Jahre als ärztlicher Direktor.

„Die guten Pflegekräfte auf der Intensivstation, mit denen lässt sich Vieles aufbauen", das äußerte er in einer Zeit, in der wir schon einmal einen großen Pflegenotstand hatten, in den 1980er Jahren. Prof. Dr. Rudolf C. Janzen war wissenschaftlich belesen. Seine breite Allgemeinbildung und Ausbildung wurde bewundert, auch gefürchtet von Kollegen und Assistenten, wenn er kritisch auf oft stundenlangen Visiten nachfragte.

Zugleich war er kritisch klinischen Studien gegenüber und immer in Sorge um das Einzelwohl der Patienten. Individualisierte Therapie wurde gelebt zu einer Zeit, in der man hiervon noch nicht sprach. Wenn ein Krankheitsbild nicht zu einer bekannten Diagnose passte, bekam der Patient eine neue Diagno- 
se, teilweise durch überraschende Wortschöpfungen, die man so in keinem Lehrbuch finden konnte, die aber seinem Zweifel gerecht wurden. Seine ihm eigene Bescheidenheit aber äußerte sich darin, dass er nie versuchte, seinen Namen in einem dieser Syndrome zu verewigen.

Er war Vorreiter und Vordenker in der neurologischen Intensivmedizin, aber auch in der Schlaganfallversorgung. 1985 war er Gründungsmitglied der ANIM (Arbeitsgemeinschaft Neurologische Intensivmedizin; heute: Deutsche Gesellschaft für NeuroIntensiv- und Notfallmedizin). Er schuf Strukturen, die bis heute fortgeführt werden. Vor allem bemühte er sich um einen Dialog zwischen den verschiedenen Partnern im Gesundheitssystem, den großen und kleinen Kliniken und den niedergelassenen Ärzten. Zeugnisse dieses Dialogs gibt es viele: der 1991 gegründete Neurowissenschaftliche Verein, die Arbeitsgruppe Schlaganfall in Hessen und die Qualitätssicherung in Hessen.

Die Unterstützung der Patientenselbsthilfeorganisation der Deutschen Myasthenie-Gesellschaft war ihm eine Herzensangelegenheit, bis in den Ruhestand.

Prof. Dr. Rudolf C. Janzen wirkte über Jahre in der Arzneimittelkommission der Ärzteschaft und beriet den gemeinsamen Bundesausschuss für Ärzte und Krankenkassen.

Er erhielt viele Ehrungen, u.a. 2005 die Ehrenplakette in Silber der Landesärztekammer Hessen für seine Tätigkeit im Lenkungsausschuss der Geschäftsstelle Qualitätssicherung, im Krankenhausausschuss und das Romberg-Glas der DGN 2006.

Im Vordergrund stand für ihn stets das gemeinschaftliche Bemühen, das Beste an Behandlung für den Patienten zu schaffen, unter Miteinbeziehung anderer Fachdisziplinen und Behandler auch im niedergelassenen Bereich. Er schickte seine Patienten zur Zweitmeinung, nie fürchtete er, Patienten zu „verlieren“ - immer suchte er den Dialog mit den Spezialisten. Seine Patienten hielten ihm und er ihnen jahrelang die Treue.

Ganz früh beschäftigte sich Prof. Dr. Rudolf C. Janzen nicht nur mit dem wissenschaftlichen Aspekt in der Intensivmedizin, sondern auch mit den menschlichen und ethischen Aspekten am Ende des Lebens. Gab es tragische Fälle auf der Intensivstation, schicksalshafte Entwicklungen bei Patienten, stellte er sich immer der Verantwortung. Er sprach mit Patienten und sprach mit Mitarbeitern, wenn diese Mühe hatten, tragische Situationen $\mathrm{zu}$ verarbeiten.

Er übernahm persönlich die Verantwortung für alles, was in seiner Klinik im Krankenhaus Nordwest geschah. In den letzten Jahren der Berufstätigkeit verzweifelte er zunehmend an der Entmenschlichung unseres Medizinbetriebs. Dokumentieren, Standards erfüllen, mit der Stechuhr arbeiten, all dies war ihm zuwider.

Das Sitzen vor Rechnern, das Verschlüsseln von Diagnosen, das Abarbeiten von Papieren zur Sicherung von Formalien, das machte Prof. Dr. Rudolf C. Janzen frustriert und traurig. Als er in den Ruhestand ging, blühte er vor unser aller Augen auf. Er konnte seine vielfältigen künstlerischen Begabungen v. a. im Malen und Zeichnen wiederbeleben.

Er übernahm aber auch erneut medizinische Verantwortung mit der Zuständigkeit für den Off-Label-Gebrauch von Medikamenten, d.h. für wirksame Substanzen ohne Zulassung auf dem Markt. Diese sollten seiner Ansicht nach den Patienten in Einzelfällen nicht vorenthalten werden. Dabei war ihm auch eine Neurologie ohne zu starke Verbindung mit der Industrie wichtig.

Seine Mitarbeiter sah er als Einzelpersonen, mit differenziertem und persönlichem Blick. Er war kein Standardlehrer, der Ausbildungspläne erfüllt, sondern ein Arzt, der als Vorbild menschlich und fachlich prägte. Hierzu gehörte auch, dass er selbstkritisch war. Wenn eine Diagnose sich als nicht richtig erwies, wurde dies nie zugedeckt, sondern war Ansporn, sich zu verbessern. Er prägte viele klinisch tätige und niedergelassene Neurologen.

Seiner Nachfolgerin machte er den Einstieg in die neue Rolle leicht. Er überließ ihr eine wohlgeordnete Klinik, mit hervorragenden Mitarbeitern, die es lieben, Neurologen zu sein, und die Offenheit und Neugierde auf Neues hatten und haben. Sein Rat war kostbar und immer da, wenn man ihn brauchte.

Wäre er zu seinem eigenen Tod befragt worden - hätte er denn gewusst, wie er vonstattenging - Prof. Dr. Rudolf C. Janzen hätte gesagt, nüchtern als Intensivmediziner, der das schon so viele, viele Male erlebt hat: „Schicksal“.

Auf sein Leben gemünzt, das prall, dicht, leidenschaftlich und reich war, passt auch sein letzter Satz am Abend des 14. Juli am Seineufer in Paris mit Blick auf das Feuerwerk: „Oh Gott, wie ist es schön hier." Prof. Dr. Rudolf C. Janzen hat seine Frau, seine Familie und Freunde, die Menschen und seinen Beruf als Neurologe geliebt.

Wenige Wochen vor seinem Tod hatte er einem langjährigen Mitarbeiter zum Einstand in den Ruhestand geschrieben, dass ihm ein Platz im Neurologenhimmel sicher sei. Prof. Dr. Rudolf C. Janzen sprach immer vom Neurologenhimmel. Wenn jemand ihn verdient hat, dann er.

„Was ein Mensch an Gutem in die Welt trägt, geht nicht verloren" (Albert Schweitzer)

Prof. Dr. med. Peter Berlit, Berlin

Prof. Dr. med. Frank Erbguth, Nürnberg

Prof. Dr. med. Rolf Hagenah, Rotenburg/Wümme

Prof. Dr. med. Uta Meyding-Lamadé, Frankfurt/Main

Dr. med. Christoph Mohs, Frankfurt/Main

Dr. rer. nat. Thomas Thiekötter, Berlin

\section{Korrespondenzadresse}

\author{
U. Meyding-Lamadé \\ Neurologische Abteilung \\ Krankenhaus Nordwest \\ Steinbacher Hohl 2-26, 60488 Frankfurt am Main, \\ Deutschland \\ meyding-lamade.uta@khnw.de
}

